\title{
Politischer Extremismus und Demokratieschutz
}

\author{
von Eckhard Jesse
}

\begin{abstract}
Die Konzeption des Extremismus steht in einem engen Zusammenhang mit der Konzeption des Demokratieschutzes. Die eine Seite ist die Kehrseite der anderen. Die Bundesrepublik Deutschland wurde und wird stark durch die Hinterlassenschaft der NS-Diktatur geprägt, ebenso, wenngleich weniger, durch die der SED. Der antiextremistische Konsens, der lange akzeptiert war, hat sich inzwischen stark abgeschwächt. Auch wenn die Extremismus- wie die Demokratieschutzkonzeption inzwischen auf Kritik stoßen, so besteht kein rationaler Grund, davon abzurücken. Im Nachgang zu der im November 2011 bekanntgewordenen Mordserie von Rechtsextremisten wird derzeit verstärkt ein Verbotsantrag gegen die NPD gefordert. Selbst für den Anhänger der streitbaren Demokratie spricht allerdings wenig dafür.
\end{abstract}

The concept of extremism is closely linked to the concept of the protection of democracy. They are two sides of the same coin. The Federal Republic of Germany has been and still is strongly determined by the legacy of the dictatorship of the Nazis as well as, although to a lesser extent, the East German SED. The anti-extremist consensus had been accepted for a long time. In the recent past, however, its acceptance has decreased considerably. Even though the concepts of extremism and of the protection of democracy currently face criticism, there is no rational reason to change course. As a result of the discovery of a series of murders committed by right-wing extremists in November 2011, calls for a ban of the far-right NPD are gaining in support. However, even to an adherent of vigilant democracy, such calls are far from convincing.

\section{Einleitende Überlegungen}

Die Traumata der Deutschen sind mit Händen zu greifen. Die Last der Geschichte lebt in aktuellen Diskursen fort. Zwei Beispiele, die ähnliche Argumentationsmuster bei gegensätzlichen Schlussfolgerungen zeigen: Die Anhänger wie die Gegner des Extremistenbeschlusses von 1972, der Grundsätze zur Fernhaltung von Extremisten aus dem öffentlichen Dienst regelte, bemühten die historischen Erfahrungen. Die einen warnten vor staatlicher Laxheit mit Blick auf die abwehrschwache Weimarer Republik, die anderen vor staatlicher Überreaktion, wenn nicht vor Exzessen, mit Blick auf das NS-Unrechtsregime. Als 1999 die Frage anstand, ob Deutschland mit anderen Staaten im Kosovo militärisch intervenieren solle, galt dies für die einen als notwendig (,nie wieder Auschwitz“), für die anderen hingegen als Sakrileg (,nie wieder Krieg“'). 
Die Anfang November 2011 bekanntgewordene Mordserie der Thüringer Rechtsextremisten, die Verhaftungen nach sich zog, rief einhellige Abscheu hervor. Die Öffentlichkeit war sich über die rückhaltlose Notwendigkeit der Aufklärung einig. Nichts dürfe vertuscht werden: nicht die Hintergründe eines möglicherweise größeren rechtsextremistischen Netzwerks, nichts über Fehler im Bereich der inneren Sicherheit im Allgemeinen und in dem des Verfassungsschutzes im Besonderen; zudem sei die Frage zu klären, wie eine solche kriminelle Gruppe 13 Jahre unentdeckt bleiben konnte und was das Neue an ihr darstelle. ${ }^{1}$ Der vom Bundestag Mitte Januar 2012 mit den Stimmen aller Fraktionen eingesetzte Untersuchungsausschuss soll dazu dienen, sowohl die Verbrechen der „Zwickauer Zelle“ als auch die Fehler der Sicherheitsbehörden aufzuklären. Die Auffassung, den Verfassungsschutz abzuschaffen, ist bei den tragenden gesellschaftlichen Kräften zwar nicht mehrheitsfähig, aber fortan war nicht mehr von Linksextremismus die Rede, sondern nur noch von Rechtsextremismus bzw. -terrorismus.

Das besondere Augenmerk, das in Deutschland auf (rechts-)extremistische Aktivitäten gerichtet ist, erklärt sich ebenso mit der leidvollen Vergangenheit wie die Konzeption der streitbaren Demokratie, die im Grundgesetz verankert ist, obwohl weder der Begriff des „Extremismus“ noch jener der „,streitbaren Demokratie" dort auftaucht. Die im Folgenden zu beantwortende Leitfrage soll daher lauten, ob heute die Konzeptionen des Extremismus und der streitbaren Demokratie noch akzeptiert sind. Daher wird zunächst die Extremismuskonzeption vorgestellt (mit der Kritik und der Gegenkritik), danach die Demokratieschutzkonzeption (auch hier unter Einschluss von Kritik und Gegenkritik). Schließlich ist vor den abschließenden Überlegungen angezeigt, den Komplex des politischen Extremismus mit dem Komplex der streitbaren Demokratie an einem Beispiel unter einer doppelten Fragestellung zusammenzuführen: Wieso ist die NPD extremistisch? Und: Soll sie verboten werden?

\section{Konzeption des Extremismus}

\section{Kennzeichen}

Der politische Extremismus zeichnet sich dadurch aus, dass er den demokratischen Verfassungsstaat ablehnt und beseitigen oder ihn einschränken will - sei

1 Pfahl-Traughber, A.: Die Besonderheiten des „,neuen“ Rechtsterrorismus. Der „Nationalsozialistische Untergrund“" in vergleichender Perspektive, in: Hirscher, G./Jesse, E. (Hg.): Extremismus in Deutschland. Schwerpunkte, Perspektiven. Vergleiche, Baden-Baden, 2012 (i.E.). 
es seine konstitutionelle Komponente (u.a.. Gewaltenteilung, Grundrechtsschutz), sei es seine demokratische (u.a. Volkssouveränität, menschliche Fundamentalgleichheit). ${ }^{2}$ Alle Varianten des Extremismus negieren demzufolge im Kern die Pluralität der Interessen, das damit verbundene Mehrparteiensystem und das Recht auf Opposition. Durch die Identitätstheorie der Demokratie, durch Freund-Feind-Stereotype, durch ein hohes Maß an ideologischem Dogmatismus und in der Regel durch ein Missionsbewusstsein geprägt, ist er vom Glauben an ein objektiv erkennbares und vorgegebenes Gemeinwohl beseelt, wähnt sich im Besitz als objektiv erkannter Gesetzmäßigkeiten, kann die Legitimität unterschiedlicher Meinungen und Interessen schwerlich dulden. Meistens ist auch die Akzeptanz von Verschwörungstheorien für extremistische Bestrebungen charakteristisch: Der eigene Misserfolg wird mit der Manipulation finsterer Mächte erklärt.

Der Begriff Extremismus wird teilweise synonym für Radikalismus und Populismus gebraucht. Das ist kritikwürdig. Extremismus ist für antidemokratische Ideologien und Bewegungen besser geeignet - weil weniger konnotativ vorbelastet - als Radikalismus, welcher bis in die erste Hälfte der 1970er Jahre dafür weite Verbreitung gefunden hat. In manchen Ländern kommt diesem Begriff geradezu ein positiver Gehalt zu. Und Populismus, eher negativ konnotiert, zielt vor allem auf die Art und Weise, wie (simpel) eine politische Kraft agi(ti)ert (gegen ,die da oben“). Eine extremistische Kraft kann populistisch sein, muss es aber nicht, eine demokratische ebenso. Radikalismus und Populismus können den Begriff des Extremismus nicht ersetzen. Es ist ebenso verwirrend, wie das vielfach geschieht, jene Richtungen, die zwischen demokratisch und extremistisch angesiedelt sind, mit diesen Termini zu bedenken.

Zuweilen legitimiert der eine Extremismus seine Existenzberechtigung mit dem Kampf gegen einen anderen. Extremisten sind - wie die Enden eines Hufeisens einander benachbart und entfernt zugleich. Extremismus ist ein Pejorativum, ein negativer Verfassungsbegriff. Daher drehen diejenigen, die als extremistisch gelten, den Spieß um und geben den Vorwurf zurück („Extremismus der Mitte“). Auf diese Weise wird der Extremismus nicht be-, sondern entgrenzt. Bei Seymour M. Lipset, der die Wendung „Extremismus der Mitte“ eingeführt hatte, war

2 Backes, U.: Extremismus in demokratischen Verfassungsstaaten. Elemente einer normativen Rahmentheorie, Opladen, 1989; Kailitz, St.: Politischer Extremismus in der Bundesrepublik Deutschland. Eine Einführung, Wiesbaden, 2004; Backes, U./Jesse, E.: Vergleichende Extremismusforschung, BadenBaden, 2005; Jaschke, H-G.: Politischer Extremismus, Wiesbaden, 2006; Böttcher, A./Mares, M.: Extremismus. Theorien - Konzepte - Formen, München, 2012. 
die Konnotation eine ganz andere. Er stellte auf die sozialen Trägerschichten ab. Der Nationalsozialismus sei ein Extremismus der Mittelklasse. ${ }^{3}$ Es gibt mit Blick auf Organisation, Ideologie und Strategie schwach und stark ausgeprägten Extremismus. Diese Abstufungen treffen ebenso für Diktaturen zu, in denen Extremisten an die Macht gelangt sind. Neben harten Formen des Extremismus finden sich zunehmend weiche, also solche, die nur einzelne Elemente des demokratischen Verfassungsstaates in Frage stellen. Die Forschung vermag dabei in Grenzfällen abweichende Ergebnisse zu erzielen. Die Existenz von Grauzonen liegt in der Natur der Sache und kann nicht dem Extremismuskonzept an sich angelastet werden. Unterschiedliche Strömungen in einer Partei - eher extremistische wie eher demokratische - sind ebenso zu berücksichtigen wie Wandlungen.

Die Formen des Extremismus sind höchst vielfältig: So kann man nach der Art der eingesetzten Mittel ebenso unterscheiden wie nach den politischen Zielen. Wer politische Gewalt systematisch einsetzt, ist ein Terrorist. Am anderen Ende des politischen Extremismus steht der Befürworter strikter Legalitätstaktik. Er bewegt sich im Rahmen der Legalität und verwirft Gewaltanwendung. Dazwischen sind jene Positionen angesiedelt, die Gewalt prinzipiell begrüßen, sie aber in der Praxis aus taktischen Gründen vorerst ablehnen. Wer Gewalt zur Durchsetzung politischer Ziele ausübt, ist ein Extremist; aber nicht jeder, der keine Gewalt anwendet, muss schon ein Anhänger des demokratischen Verfassungsstaates sein.

Was die politische Zielsetzung angeht, so wird gemeinhin zwischen dem Linksund dem Rechtsextremismus differenziert. Mit Linksextremismus ist jene Variante des Extremismus gemeint, die alle Übel des „Systems“ in der Struktur der „kapitalistischen Klassengesellschaft“ sieht („Kommunismus“) oder die den Staat generell ablehnt (,Anarchismus"). Unter Rechtsextremismus versteht man einen häufig mit Rassismus verbundenen Nationalismus. Während der Extremismus von rechts das Prinzip menschlicher Fundamentalgleichheit ablehnt, stellt der Extremismus von links dies - jedenfalls in der Theorie - nicht in Frage, verabsolutiert Gleichheit geradezu (auf Kosten der Freiheit). Obwohl rechts- und linksextremistische Bestrebungen, die sich jeweils wieder in unterschiedliche Richtungen aufspalten, einander heftig bekämpfen, benötigen ihre Repräsentanten sich gegenseitig: Die Warnung vor dem Rechtsextremismus („Antifaschis-

3 Vgl. Lipset, S.M.: Der „Faschismus“, die Linke, die Rechte und die Mitte, in: Kölner Zeitschrift für Soziologie und Sozialpsychologie 11 (1959), 401-444. Der Beitrag wurde vielfältig nachgedruckt. 
mus") nimmt bei Linksextremisten einen überdimensionalen Raum ein. Vice versa gilt das ebenso, freilich nicht im gleichen Maße. Auf diese Weise versucht jedoch die eigene Richtung die Existenzberechtigung nachzuweisen. Allerdings ist „Antifaschismus“ weitaus wirkungsmächtiger als „Antikommunismus“. Der religiös ausgerichtete Fundamentalismus, etwa in Form des Islamismus, gilt als eine eigenständige Spielart des Extremismus, jenseits von rechts und links. Er strebt einen „Gottesstaat“ an. In Deutschland spielte diese Variante des Extremismus in der Vergangenheit eine eher untergeordnete Rolle. Es gibt keine Homogenität „des“ Rechts-, „des“ Links- und „des“ islamistischen Extremismus. Dies ist Ausdruck eines hohen ideologischen Dogmatismus. Allein der Trotzkismus umfasst eine Vielzahl an Richtungen, die alle Anspruch auf die ,reine Lehre" erheben.

Der Begriff des Extremismus hat dem Sinne nach eine lange historische Tradition, die bis auf Platon und Aristoteles zurückreicht. Mit seiner „Mischverfassung" hat Aristoteles in gewisser Weise den Grundstein für die Theorie des demokratischen Verfassungsstaates gelegt. Der Begriff Extremismus tauchte bereits - 1646 - beim Calvinisten Ludwig Camerarius auf, der ihn auf die Jesuiten anwandte, wurde aber nicht rezipiert. Der Leipziger Philosoph Wilhelm Traugott Krug hatte 1838 in einem Handwörterbuch den Begriff erstmals vergleichend gebraucht - für den (rechten) „Absolutismus“ wie den (linken) „Radikalismus“. Im Zuge der Russischen Revolution fand der Terminus v.a. in England und Frankreich weite Verwendung, zunächst beschränkt auf den Extremismus von links. Von den 1920er Jahren an spielte Extremismus in der wissenschaftlichen Forschung eine gewisse Rolle, ohne aber die Bedeutung des Terminus „Totalitarismus“ zu erreichen, obwohl älteren Ursprungs. Uwe Backes hat in einer ideengeschichtlichen Grundlagenstudie die Entfaltung des Begriffs erhellt. ${ }^{4}$

Nach dem Zweiten Weltkrieg stellte der amerikanische Soziologe Seymour M. Lipset den Gegensatz von Pluralismus und Monismus als zentral heraus. ${ }^{5}$ In Deutschland war es der Kölner Soziologe Erwin K. Scheuch, der an dessen Forschungen anknüpfte und sie in empirischen Analysen weiterentwickelte. ${ }^{6}$ Hans-

4 Backes, U.: Politische Extreme. Eine Wort- und Begriffsgeschichte von der Antike bis zur Gegenwart, Göttingen, 2006.

5 Lipset, S.M.: Political Man. The Social Basis of Politics, New York, 1960; ders./Raab, E.: The Politics of Unreason. Right-Wing Extremism in America, 1790-1977, Chicago, 1978.

6 Scheuch, E.K.: Politischer Extremismus in der Bundesrepublik, in: Löwenthal, R./Schwarz, H.-P. (Hg.): 25 Jahre Jahre Bundesrepublik Deutschland - eine Bilanz, Stuttgart, 1974, 433-469. 
Dieter Klingemann und Franz U. Pappi unterschieden zwischen einer Ziel- und einer Mitteldimension. ${ }^{7}$ Damit war gerade die empirische Sozialwissenschaft in den Anfängen der Extremismusforschung dominierend. Die 1987 vertretene Auffassung, die Extremismusforschung sei ein „Stiefkind der Politikwissenschaft“", trifft heute zum Teil immer noch zu, da etwa Kommunismus-, Rechtsextremismus- und Islamismusforschung häufig nicht als Einheit gelten, sondern nach wie vor separat erörtert werden. Die wegweisende Studie von Norberto Bobbio differenziert - ähnlich wie Klingemann/Pappi - nicht nur zwischen links und rechts (Kriterium: Gleichheit), sondern auch zwischen extrem und gemäßigt (Kriterium: Freiheit). Der Autor gelangt damit zu vier politischen Richtungen: „extreme Linke“, „linke Mitte“, „rechte Mitte“, ,extreme Rechte“.9

Neben dem organisierten Extremismus (etwa in Parteien) ist der sich oft in Gewalt entladende subkulturelle Extremismus zu erwähnen. Während der Großteil der Gewalttaten von rechtsaußen eher spontan geschieht, zeichnet sich die Szene linksaußen durch ein weit höheres $\mathrm{Ma} ß$ an Planungsintensität aus. Unterschiede in der Tatspezifik (der prozentuale Anteil der Körperverletzungen ist rechtsaußen höher, derjenige der Landfriedensbrüche linksaußen) gehen z.T. auf die unterschiedliche soziale Charakteristik der Akteure zurück: niedrigeres Durchschnittsalter, niedriger Bildungsstand, stärkeres männliches Übergewicht und größere Neigung zu körperlicher Gewalt rechtsaußen. Während die rechte Gewalt eher expressiv ist (Gewalt als Selbstzweck), ist die von links stärker instrumentell bestimmt (Gewalt als Mittel zum Zweck). ${ }^{10}$

Extremistische Kräfte suchen den Eindruck hervorzurufen, der demokratische Verfassungsstaat müsse vor den drängenden Problemen der Gegenwart kapitulieren - sei es Arbeitslosigkeit, sei es Zuwanderung. Die politische Kultur in Deutschland scheint so gefestigt, dass die Gefahr extremistischer Erfolge auf längere Dauer kleiner wurde, mag auch die Zunahme der Liberalität - ein charakteristisches Indiz des Wandels der politischen Kultur - insofern ambivalent sein, als sie Erosionstendenzen gegenüber dem Extremismus begünstigt, zumal dem von links. Aber eine offene Gesellschaft wie die der Bundesrepublik ist dadurch in ihrer Stabilität nicht gefährdet.

7 Klingemann, H.D./Pappi, F.U.: Politischer Radikalismus. Theoretische und methodische Probleme der Radikalismusforschung, dargstellt am Beispiel einer Studie anlässlich der Landtagswahl 1970 in Hessen, München/Wien, 1972.

8 Backes, U./Jesse, E.: Extremismusforschung - ein Stiefkind der Politikwissenschaft, in: Michalka, W. (Hg.): Extremismus und streitbare Demokratie, Stuttgart, 1987, 9-28.

9 Bobbio, N.: Rechts und Links. Gründe und Bedeutungen einer politischen Unterscheidung, Berlin, 2006.

10 Backes, U./Mletzko, M./Stoye, J.: NPD-Wahlmobilisierung und politisch motivierte Gewalt, Köln, 2010. 


\section{Kritik und Gegenkritik}

Die Kritik an der Forschung zum Extremismus reicht von Detail- bis zu Fundamentalkritik. So heißt es, Analysen zum Extremismus seien unterkomplex, staatszentriert, ideologiegesättigt, auf Analogien höchst unterschiedlicher Phänomene bedacht und stark an der als repressiv geltenden streitbaren Demokratie orientiert, also auf den Erhalt der - idealisierten - Mitte und des status quo. Vor allem die vergleichende Forschung zum Extremismus - die das Äquidistanzgebot bejaht - ruft viele Einwände vor, ist doch der antiextremistische Konsens heute in Deutschland nicht mehr so selbstverständlich wie früher. Gewiss muss jede normative Vorgehensweise berücksichtigen, ob nicht der demokratische Staat erst Probleme verursacht, durch die der Extremismus anwächst. Die Wechselwirkungen zwischen Extremismus und Demokratie werden in der Tat häufig vernachlässigt.

Im Folgenden geht es vor allem darum, die Position des Kölner Politikwissenschaftlers Christoph Butterwegge, eines scharfen Kritikers des Extremismusbegriffs, näher unter die Lupe zu nehmen. Er war 2012 einer der von der Partei Die Linke ins Auge gefassten Kandidaten für das Amt des Bundespräsidenten. In einem Beitrag aus dem Jahre 2011 heißt es bei ihm: „Wer nach zwei Seiten zugleich schaut, hat nie gezielt und trifft kaum seinen eigentlichen Gegner. Wer gleichermaßen nach links- und rechtsaußen starrt, verliert die Entwicklung in der politischen Mitte als mögliche Hauptbedrohung für die Demokratie zwangsläufig aus dem Blick." ${ }^{11}$ Diese Formulierungen sind verräterisch: Was heißt ,eigentlicher Gegner"? Und wieso verliert, wer nach links- und rechtsaußen schaut, ,die Mitte“ aus dem Blick? Und weswegen ist diese - „die Mitte“ - die „mögliche Hauptbedrohung“? Tatsächlich spielt der vage Begriff der „Mitte“ in der Extremismusforschung keine Rolle. Es geht um die Akzeptanz des demokratischen Verfassungsstaates.

Christoph Kopke und Lars Rensmann sehen dies anders: Bei ihnen heißt es: „Politische Orientierungen erscheinen [...] völlig beliebig, links und rechts werden gleichgesetzt und austauschbar, sofern man außerhalb der willkürlich gesetzten Mitte steht. Der ,Extremist‘ wird schlicht konstruiert als ,verhaltensauffälliger' Außenseiter, der einer hermetischen Programmatik und einem teilungs-

11 Butterwegge, Ch.: Linksextremismus = Rechtsextremismus? Über die Konsequenzen einer falschen Gleichsetzung, in: Birsl, U. (Hg.): Rechtsextremismus und Gender, Opladen, 2011, 34. 
unwilligen Machtanspruch folgt.“12 Begriffe wie ,Mitte“ und ,verhaltensauffällig' werden in Anführungszeichen gesetzt, als stammten die Begriffe von Extremismusforschern. Diese rechtfertigen entgegen stereotypen Insinuationen keineswegs unkritisch die ,herrschende Politik“. Der demokratische Verfassungsstaat ist vielfältig gefährdet. Jeder Rechtsextremist ist ein Antidemokrat, aber nicht jeder Antidemokrat ein Rechtsextremist, jeder Stalinist ein Linksextremist, doch nicht jeder Linksextremist ein Stalinist.

Der Begriff der Äquidistanz provoziert besonders viele Proteste. Selbstverständlich ist damit nicht gemeint, jede extremistische Spielart sei gleichweit vom demokratischen Verfassungsstaat entfernt. Doch müssen für alle Varianten dieselben Beurteilungsmaßstäbe gelten. Butterwegge schüttet das Kind mit dem Bade aus. „Man kann unter dem Oberbegriff ,Krankheiten“ auch Hautkrebs und Hühneraugen miteinander vergleichen; dies wird aber kein seriöser Mediziner tun. “13 Fluchtpunkt der Überlegungen des Autors und anderer prinzipieller Gegner der Extremismusforschung ist die Behauptung, die rechte und die linke Seite des politischen Spektrums werde gleichgesetzt. Butterwegge spricht bereits im Titel von einer „,falschen Gleichsetzung“. „Extremismustheoretiker setzen Linksund Rechtsextremismus nämlich mehr oder weniger explizit gleich." Ein Vergleich, der „Feuer mit Wasser vergleicht und beide womöglich auch noch gleichsetzt ", ${ }^{14}$ bringe keinen Erkenntnisgewinn. Das ist nun gleich in zweierlei Hinsicht nicht stimmig. Zum einen geht es ganz und gar nicht um Gleichsetzung, sondern um Vergleiche mit Blick auf Gemeinsamkeiten und Unterschiede. Zum anderen wird nicht Feuer mit Wasser verglichen. Denn die Phänomene weisen bei aller Gegensätzlichkeit strukturelle Analogien auf. Aus der Tatsache, dass der Linksextremismus den Rechtsextremismus bekämpft (und vice versa), folgt nicht, es gäbe keine Feindschaft ohne Nähe.

Im übrigen schließt der Vorwurf der Gleichsetzung einen anderen erhobenen Vorwurf prinzipiell aus. So heißt es bei Butterwegge, Extremismusforscher grenzten Linke als potentielle Verbündete aus, hätten aber wenig Berührungsängste gegenüber ultrarechten Kräften und Positionen. Von da aus ist es nicht weit bis zum Vorwurf, die Extremismusforscher seien „staatsfixiert“, was „zu den Hauptkennzeichen des Rechtsextremismus in Deutschland, nicht aber des

12 Kopke, Ch./Rensmann, L.: Die Extremismus-Formel. Zur politischen Karriere einer wissenschaftlichen Ideologie, in: Blätter für deutsche und internationale Politik 45 (2000), 1452.

13 Butterwegge, Ch., a.a.O., 2011, 36.

14 Ebd., 34. 
Linksradikalismus zählt.“'15 Tatsächlich geht es der Extremismusforschung darum, den Extremismus als Widerpart des demokratischen Verfassungsstaates anzusehen. Rechtsextremisten sind jedoch keine Repräsentanten des demokratischen Verfassungsstaates. ${ }^{16}$

Der verbreitete Eindruck, ein normativer Ansatz stünde einem empirischanalytischen Ansatz gegenüber, ist so nicht triftig. Gero Neugebauer und Richard Stöss warnen in ihrer instruktiven Studie über die PDS davor, den Begriff der Partei „mit einem normativen Demokratiegebot [zu] überfrachten“. ${ }^{17}$ Bei der Analyse des parteiförmigen Rechtsextremismus dagegen bekennt sich Stöss durchaus zu einem normativen Gebot, nämlich dem des Antifaschismus - „als politische Norm und als pädagogisches Ziel“" ${ }^{18}$ Tatsächlich - und das ist wohl der Kern der Kontroverse - liegt der einen Auffassung ein antiextremistischer Ansatz zugrunde, der anderen ein - jedenfalls verdeckt - antifaschistischer. Wer die Extremismusforschung unvoreingenommen sichtet, kann die Behauptung des Berliner Rechtsextremismus- und Parteienforschers Richard Stöss, der weniger plump als Butterwegge argumentiert, wohl kaum mehr aufrechterhalten, der „eigentliche Gebrauchswert des Extremismuskonzepts“ liege darin, dass die „Tür für subjektive Werturteile weit offen steht “. ${ }^{19}$ Träfe dies zu, müsste Stöss konsequenterweise Abstand vom Begriff des Rechtsextremismus nehmen, den er zahlreichen seiner instruktiven Arbeiten zugrunde gelegt hat.

Auch wenn die Zahl der Kritiker des Extremismusansatzes beträchtliche Ausmaße angenommen hat ${ }^{20}$, dürfte die Überlegung, ,ob der Begriff [des Extremismus] nicht hinfällig geworden ist ${ }^{\text {‘21 }}$, keineswegs haltbar sein. Wer den „Berliner Mauerfall und seine Folgen geradezu als Waterloo für die Extremismus- und Totalita-

15 Ebd., 30 .

16 Zur überzeugenden Kritik an Butterwegge vgl. auch Pfahl-Traughber, A.: Kritik der Kritik der Extremismus- und Totalitarismustheorie. Eine Auseinandersetzung mit den Einwänden von Christoph Butterwegge, in: ders.: (Hg): Jahrbuch für Extremismus- und Terrorismusforschung 2009/2010, Brühl, 2010, 61-86.

17 Neugebauer, G./Stöss, R.: Die PDS. Geschichte, Organisation, Wähler, Konkurrenten, Opladen, 1996, 13.

18 So Stöss, R.: Die extreme Rechte in der Bundesrepublik Deutschland. Entwicklung - Ursachen - Gegenmaßnahmen, Opladen, 1989, 244.

19 Ders.: „Extremistische Parteien“ - Worin besteht der Erkenntnisgewinn?, in: Aus Politik und Zeitgeschichte B 47/2008, 7.

20 Zuletzt etwa: Forum für kritische Rechtsextremismusforschung (Hg.): Ordnung. Macht. Extremismus. Effekte und Alternativen des Extremismus-Modells, Wiesbaden, 2011.

21 Neugebauer, G.: Einfach war gestern. Zur Strukturierung der politischen Realität in einer modernen Gesellschaft, in: Aus Politik und Zeitgeschichte B 44/2010, 9. 
rismustheorie $^{\text {‘22 } 2}$ ansieht, muss mit Blindheit geschlagen sein. Der friedliche Verlauf der weltweiten Systemwechsel widerlegt die Extremismusforschung keineswegs. Ihr politische Interessengeleitetheit $\mathrm{zu}$ unterstellen, fällt auf die Urheber zurück.

Trotzdem gibt es Defizite. Die Annahme, der organisierte Extremismus sei gut erforscht, bedarf der Relativierung. Der Vergleich zwischen rechtsextremistischen Bestrebungen in verschiedenen Staaten ist weit verbreitet und bringt zahlreiche erhellende Ergebnisse (etwa zu Einstellungsmustern, Entstehungsbedingungen und Gefährdungspotenzialen) mit sich. ${ }^{23}$ Deutlich geringer ist die Zahl der Studien zum Linksextremismus in Europa. ${ }^{24}$ Erst recht fehlt es an Ländervergleichen unter Einbeziehung aller Varianten. ${ }^{25}$ Ein beträchtlicher Teil der Forschung sieht das Feld des politischen Extremismus nicht als Einheit an, will partout die eine Spielart des Extremismus nicht mit der anderen vergleichen. Das gilt keineswegs nur für Deutschland.

Die Forschung zur Messung extremistischer Einstellungen ist weit verbreitet. Allerdings hängt die Überzeugungskraft der Ergebnisse wesentlich von der Art der Fragestellung ab. Bei ,weichen“ Indikatoren geht das einschlägige Einstellungspotential nach oben, bei „harten“ Indikatoren nach unten. Die Forschung zum „Rechtsaußenpotenzial“ ist deutlich besser ausgeprägt als die zum „Linksaußenpotenzial“. In jüngster Zeit hat vor allem Viola Neu den Versuch unternommen, für beide Dimensionen eine Extremismusskala zu entwickeln. ${ }^{26}$

Unübersehbar groß ist die Zahl der Studien zu rechtsextremistischen Skinheads, NS-affinen Gruppen und Gewalttätern von rechts. ${ }^{27}$ Hingegen gibt es nur wenige Analysen zum linksautonomen Milieu $^{28}$ und der Antifa-Gewalt. Erst in jüngster

22 Butterwegge, Ch., a.a.O., 2011, 35.

23 Arzheimer, K.: Die Wähler der extremen Rechten 1980-2002, Wiesbaden 2008; Backes, U./Moreau, P. (Hg.): The Extreme Right in Europe. Current Trends and Perspectives, Göttingen, 2012.

24 Backes, U./Moreau, P. (Hg.): Communist and Post-Communist Parties in Europe, Göttingen 2008; Mannewitz, T.: Linksextremistische Parteien in Europa nach 1990. Ursachen für Wahlerfolge und misserfolge, Baden-Baden, 2012.

25 Jesse, E./Thieme, T. (Hg.): Extremismus in den EU-Staaten, Wiesbaden, 2011.

26 Vgl. etwa $\mathrm{Neu}, \mathrm{V}$ : Rechts- und Linksextremismus in Deutschland. Wahlverhalten und Einstellungen, Sankt Augustin, 2009.

27 Etwa: Thein, M.: Wettlauf mit dem Zeitgeist. Der Neonazismus im Wandel. Eine Fallstudie, Göttingen 2010; Schedler, J./Häusler, J. (Hg.): Autonome Nationalisten. Neonazismus in Bewegung, Wiesbaden, 2011.

28 Hoffmann, K.D.: „Rote Flora“. Ziele, Mittel und Wirkungen eines linksautonomen Zentrums in Hamburg, Baden-Baden, 2011. 
Zeit wurde der Interaktionsdynamik links- und rechtsextremer Gruppen Aufmerksamkeit geschenkt, ${ }^{29}$ obgleich viele Entwicklungen nur unter Berücksichtigung der Wechselwirkungen und Interdependenzen extremistischer Akteure und ihrer „Umwelt“ (etablierte Parteien, Medien, Bürgergesellschaft etc.) angemessen zu interpretieren sind. Weitere Inkonsistenzen und Desiderate ließen sich leicht aufzählen: Studien zum intellektuellen Rechtsextremismus gibt es zuhauf (nicht immer mit der nötigen wissenschaftlichen Distanz), ${ }^{30}$ jedoch nicht zum intellektuellen Linksextremismus. Der Grund liegt schwerlich darin, dass die eine Form erfolgreich, die andere erfolglos ist.

\section{Konzeption des Demokratieschutzes}

\section{Kennzeichen der streitbaren Demokratie}

Das Konzept der streitbaren Demokratie, das mit den Namen von Karl Loewenstein und Karl Mannheim verbunden ist,${ }^{31}$ liegt dem Grundgesetz zugrunde. Das Schicksal der Weimarer Republik warf die Frage auf, ob die Demokratie nicht Mechanismen zum eigenen Schutz vorsehen kann und soll. Die Konzeption der streitbaren Demokratie will die Hilflosigkeit der relativistisch geprägten Demokratie des Weimarer Typs überwinden. Ihr zentraler Gedanke ist die Vorverlagerung des Demokratieschutzes in den Bereich des legalen politischen Handelns. Der demokratische Verfassungsstaat soll sich seiner Gegner nicht erst erwehren können, wenn diese Strafgesetze übertreten. Die Demokratie Weimarer Prägung konnte gegenüber solchen Gruppierungen nichts unternehmen, da sie sich (überwiegend) keines Verstoßes gegen Gesetze schuldig machten.

Für alle Varianten der streitbaren Demokratie gilt, dass sie drei Charakteristika umfassen: (1) die Wertgebundenheit, (2) die Abwehrbereitschaft und (3) die Vorverlagerung des Demokratieschutzes, wobei dieser letzte Punkt eine Präzisierung des zweiten darstellt. Mit Wertgebundenheit ist gemeint, dass der Verfassungsstaat eine Wertordnung zur Grundlage hat, die er nicht zur Disposition gestellt wissen will. Zum demokratischen Minimalkonsensus zählen insbesonde-

29 Vgl. Backes, U./Mletzko, M./Stoye, J., a.a.O., 2010.

30 Heni, C.: Salonfähigkeit der Neuen Rechten. ,Nationale Identität', Antisemitismus und Antiamerikanismus in der politischen Kultur der Bundesrepublik 1970-2005: Henning Eichberg als Exempel, Marburg, 2007.

31 Loewenstein, K.: Militant Democracy and Fundamental Rights (I + II), in: American Political Science Review 31 (1937), 417-432, 638-658; Mannheim, K.: Diagnose unserer Zeit. Gedanken eines Soziologen, Zürich, 1951. Das Buch erschien 1941 zunächst auf Englisch; der erstmalige Gebrauch des Begriffs der „streitbaren Demokratie“ (17) geht auf den Übersetzer Mannheims zurück. 
re die Menschenrechte. Das Grundgesetz kennt ein „Ewigkeitsgebot“. Art. 79 Abs. 3 GG lautet bekanntlich: „Eine Änderung dieses Grundgesetzes, durch welche die Gliederung des Bundes in Länder, die grundsätzliche Mitwirkung der Länder bei der Gesetzgebung oder die in den Artikeln 1 und 20 niedergelegten Grundsätze berührt werden, ist unzulässig.“ Diese Werte und institutionellen Verfahrensnormen sind die Legitimationsbasis der Streitbarkeit.

Zur Abwehrbereitschaft gehört die Verteidigung des demokratischen Verfassungsstaates gegenüber extremistischen Positionen. Art. 9 Abs. 2 GG sieht die Möglichkeit des Vereinigungsverbots vor, Art. 21 Abs. 2 die des Parteienverbots. Art. 18 erlaubt die Verwirkung der Grundrechte, um drei wesentliche Maßnahmen des präventiven Demokratieschutzes zu nennen. Demokratie ist also weder mit der Lehre der Volkssouveränität noch mit formal verstandenen Prinzipien wie Pluralismus und Toleranz identisch. Als Vorverlagerung des Demokratieschutzes gilt der Sachverhalt, dass der demokratische Verfassungsstaat es sich vorbehält, nicht erst bei einem Verstoß gegen (Straf-)Gesetze zu reagieren. Der politische Extremismus kann bereits im Bereich legalen, aber verfassungsfeindlichen Handelns gestört werden. Der Zusammenhang von Wehrhaftigkeit und Werthaftigkeit liegt auf der Hand. Ein Staat, der auf unveränderbaren Werten ruht, muss abwehrbereit sein. Und wer Abwehrbereitschaft bejaht, kommt ohne Wertgebundenheit nicht aus.

Allerdings wirft die Vorverlagerung des Demokratieschutzes für die zu gewährleistende Liberalität des Staates gravierende Probleme auf. Wird nicht gerade dadurch, dass die Legalität des Verhaltens keineswegs der einzige Maßstab für die Beurteilung ist, die Demokratie unterminiert? Spielt man auf diesem Wege Legalität und Legitimität gegeneinander aus? Wie kann sich eine im Ruch der Verfassungsfeindlichkeit stehende Organisation überzeugend gegen den Vorwurf wehren, sie tarne sich? Muss ein Rechtsstaat sich nicht ausschließlich an den Gesetzen orientieren? Fördert die streitbare Demokratie, wenn auch unbeabsichtigt, vielleicht gerade $M c$ Carthyismus?

Wie beim Extremismus gibt es beim Demokratieschutz eine weiche, stärker auf das Opportunitätsprinzip abstellende, und eine harte Form, die sich eher am Legalitätsprinzip orientiert. Die junge Demokratie reagierte zunächst verunsichert. 1952 wurde die Sozialistische Reichspartei (SRP) verboten, 1956 die Kommunistische Partei Deutschlands (KPD). Die Bundesrepublik wollte mit den beiden Verboten Exempel statuieren. Diese waren nicht nur rechtmäßig, sondern wohl auch zweckmäßig. Die Urteile des Gerichts, die den Freiraum für das Wir- 
ken politischer Parteien großzügig auslegten, zeichneten sich in hohem Maße durch Zurückhaltung und Liberalität aus. ${ }^{32}$

Bis zum Inkrafttreten des Vereinsgesetzes im Jahr 1964 wurde von der Bestimmung des Art. 9 Abs. 2 GG rege Gebrauch gemacht: Es kam allein zu 328 Verbotsverfügungen. Die hohe Zahl erklärt sich damit, dass Verbote gegen dieselbe Organisation auf der Landesebene erfolgten - zum Teil sogar auf der Ebene der Regierungspräsidien. Zudem orientierte sich die Exekutive seinerzeit am Legalitätsprinzip. Die Ausschaltung von insgesamt 64 Vereinigungen (40 links- und 24 rechtsextremistischen) aus dem politischen Leben hat der Liberalität wohl eher geschadet, die innere Sicherheit kaum gestärkt. Seit 1964 ist vom Bundesministerium des Innern eine Vielzahl Verbotsverfügungen ergangen. ${ }^{33}$ Es handelt sich ausschließlich um ausländische Vereinigungen (etwa den islamistischen „Kalifatsstaat" im Jahr 2001) oder um rechtsextremistische deutsche (u.a. Wehrsportgruppe Hoffmann 1980, Wiking Jugend 1994, Blood \& Honour Division Deutschland, White Youth 2000). Linksextremistische - inländische - Vereinigungen, wie etwa die „Rote Hilfe“, wurden nicht verboten. Zusätzlich gab es zahlreiche Verbote von Gruppierungen, die nur in einem Land aktiv waren.

Die vier Anträge auf Grundrechtsverwirkung gemäß Art. 18 GG - 1960 gegen Otto Ernst Remer, 1969 gegen Gerhard Frey, 1992 gegen Heinz Reisz und Thomas Dienel - scheiterten sämtlich. Sie wurden wegen offenkundiger Aussichtslosigkeit von der Bundesregierung nicht weiter verfolgt. Da für den demokratischen Verfassungsstaat die Herausforderung von organisierten Kräften ausgeht, ist der Artikel nicht effizient und wohl kein Zeichen demokratischer Souveränität. $^{34}$

Vom Jahre 2000 an wurden Präventionsprogramme gegen Rechtsextremismus und dessen Gewalt durch die jeweiligen Bundesregierungen gefördert. Seit der christlich-liberalen Regierung 2009 gibt es solche nun auch gegen Linksextremismus und Islamismus, freilich in weitaus bescheidenerem Maße. Dieser Umstand löste heftige Kritik bei den Oppositionsparteien aus, zumal dann, als be-

32 Kluth, H.: Die KPD in der Bundesrepublik. Ihre politische Tätigkeit und Organisation 1945-1956, Köln, 1959; Hansen, H.: Die Sozialistische Reichspartei. Aufstieg und Scheitern einer rechtsextremen Partei, Düsseldorf, 2007.

33 Einzelheiten finden sich bei Gerlach, J.: Die Vereinsverbotspraxis der streitbaren Demokratie. Verbieten oder Nicht-Verbieten, Baden-Baden, 2012.

34 Vgl. Jesse, E.: Grenzen des Demokratieschutzes in der offenen Gesellschaft. Das Gebot der Äquidistanz gegenüber politischen Extremismen, in: Backes, U./Jesse, E. (Hg.): Gefährdungen der Freiheit. Extremistische Ideologien im Vergleich, Göttingen, 2006, S. 393-420. 
kannt wurde, dass die Bundesministerien die Vergabe der Gelder im Kampf gegen jede Art des Extremismus von einer Bekundung abhängig machte, wonach die Träger der Projekte eine „Demokratieerklärung“ („Extremismusklausel“) unterschrieben und die Akzeptanz der freiheitlichen demokratischen Grundordnung auch für die mit ihnen zusammenarbeitenden Initiativen bestätigten. ${ }^{35}$ Anders als den Jahren zuvor rückte damit der früher vernachlässigte Linksextremismus zeitweilig stärker in den Blickwinkel der Öffentlichkeit. ${ }^{36}$

\section{Kritik und Gegenkritik}

Kein Komplex der streitbaren Demokratie löste eine solche Auseinandersetzung aus, wie der zum Teil in irrationalen Formen geführte Streit um die Fernhaltung von Extremisten aus dem öffentlichen Dienst. Insbesondere bei vielen Gegnern des Extremistenbeschlusses - ein weitaus treffenderer Terminus als jener des „Radikalenerlasses“ - herrschten recht simple argumentative Strickmuster vor. Eine indirekte Folge des Extremistenbeschlusses war die alsbald ins Schussfeld der Kritik geratene „Regelanfrage“. In weiten Teilen der politischen Parteien und der öffentlichen Meinung setzte sich die Auffassung durch, der Extremistenbeschluss sei ein Fehler gewesen. Dessen von heftiger Kritik begleitete Geschichte war eine Geschichte seiner ständigen Rücknahme. ${ }^{37}$ Im Zuge des Extremistenbeschlusses von 1972 setzte eine heftige Diskussion über Sinn und Unsinn, Chancen und Gefahren der streitbaren Demokratie ein. Dabei überwogen klar die Kritiker der streitbaren Demokratie, wurde doch gemutmaßt, es komme zu einer unvertretbaren Einschränkung der Freiheitsrechte. Schlagworte wie „Duckmäusertum“ und „Gesinnungsschnüffelei“ spielten in der Diskussion eine große Rolle, ohne dafür in der Praxis substantielle Gründe zu finden. Wegen mangelnder Verfassungstreue wurden nicht viel mehr als 1.000 Bewerber abgelehnt, die in der Regel einer extremistischen Organisation angehört hatten (wie etwa der DKP).

35 Vgl. die Dokumentation der Kontroverse (mit Beiträgen von Dorothee Bär, Florian Bernschneider, Ulla Jelpke, Monika Lazar, Sönke Rix): Der Streit um die „Extremismusklausel“, in: Backes, U./Gallus, A./Jesse, E. (Hg.): Jahrbuch Extremismus \& Demokratie, Bd. 23, Baden-Baden, 2011, 108-126.

36 Die Bundeszentrale für politische Bildung veröffentlichte jüngst - erstmals nach vielen Jahren - ein Buch über den Linksextremismus. Vgl. Dovermann, U. (Hg.): Linksextremismus in der Bundesrepublik Deutschland, Bonn, 2011.

37 Braunthal, G.: Politische Loyalität und Öffentlicher Dienst. Der "Radikalenerlass" von 1972 und die Folgen, Marburg, 1992. 
Nicht immer war leicht erkennbar, dass sich die Kritik dabei aus zwei ganz unterschiedlichen Quellen speiste, der wertrelativistischen und der antifaschistischen. Die wertrelativistische Position leugnet die Auffassung, es könne verfassungsfeindliche Ziele geben. Für diese Richtung stehen Claus Leggewie und Horst Meier. ${ }^{38}$ „Das Prinzip streitbarer Demokratie ist nicht zu liberalisieren, eben weil in ihm die Illiberalität zur Struktur gerann. Der diskutable Kern der Sache ist mit der Frage formuliert, auf welche Weise demokratische Selbstverteidigung in rechtsstaatlichen Formen zu konzipieren sei. Dabei gibt es zur Gewaltgrenze keine vernünftige und rechtsstaatliche rationalisierbare Alternative. Es ist daher an der Zeit, sich in der politisch längst konsolidierten Bundesrepublik von einer obsolet gewordenen ,streitbaren Demokratie' Ideologie zu emanzipieren und sich schlicht für Demokratie zu entscheiden. ${ }^{\text {‘39 }}$ Allerdings halten die Autoren ihre Position nicht konsequent durch. So plädieren sie für das Verbot einer Partei, die ausdrücklich an die Ziele der NSDAP anknüpft. Diese eng gefasste Bestimmung gilt ,,als gesinnungsbezogenes Meinungs- und Organisationsdelikt einer unausgewogenen , antinazistischen Grundordnung “، 40

Die andere Kritik an der streitbaren Demokratie stört nicht deren Militanz, sondern die Richtung, gegen die sie sich wendet. Sie versteht sich als ,,antifaschistisch“". ${ }^{41}$ Die Demokratie werde nur von einer Seite bedroht und es sei notwendig, gegen diese Militanz vorzugehen („Faschismus ist keine Meinung, sondern ein Verbrechen“). Die Partei Die Linke, die sich diese Parole zu Eigen macht, will schon seit Jahren eine ,antifaschistische Klausel“ im Grundgesetz und in den Landesverfassungen verankern.

Beide Positionen sind nicht einleuchtend: Die eine fällt in das für überwunden geglaubte Weimarer Verfassungsrechtsdenken zurück, die andere interpretiert das Grundgesetz einseitig antifaschistisch. Es gibt extremistisches Denken, und dieses findet sich nicht nur auf einer Seite des politischen Spektrums. Freilich mutet das Plädoyer für den Verzicht auf Streitbarkeit und den Verzicht auf eine

38 Leggewie, C./Meier, H.: Republikschutz. Maßstäbe zur Verteidigung der Demokratie. Mit zwei Exkursen von Alexander Molter und Wolfgang Stenke, Reinbek, 1995.

39 Dies.: Die Berliner Republik als Streitbare Demokratie? Vorgezogener Nachruf auf die freiheitliche demokratische Grundordnung, in: Blätter für deutsche und internationale Politik 37 (1992), 603 (Hervorhebung im Original).

40 Dies., Republikschutz, a.a.O., 319.

41 Kutscha, M.: Verfassung und „streitbare Demokratie”. Historische und rechtliche Aspekte der Berufsverbote im öffentlichen Dienst, Köln, 1979. 
Verbotsmentalität ${ }^{42}$ weitaus sympathischer an als die Einseitigkeit antifaschistischer Lehren, die zum Teil in eine Rechtfertigung linksextremistischer Maximen münden können.

Die in der Bundesrepublik geltende Konzeption der streitbaren Demokratie unterscheidet sich sowohl von der formalen Demokratie des Weimarer Musters als auch von einem Demokratietyp, der nach außen streitbare Elemente meidet, faktisch aber jedenfalls teilweise für ihre Geltungskraft indirekt Sorge trägt, etwa dadurch, dass die Bekämpfung in einer „diskreteren", jedenfalls weniger auffälligen Art geschieht. Insofern sollte der Begriff des deutschen Sonderwegs nicht umstandslos verwendet werden. ${ }^{43}$

\section{NPD und NPD-Verbotsantrag}

\section{Extremistischer Charakter der NPD?}

Die 1964 gegründete NPD - seinerzeit weithin deutschnational und antikommunistisch orientiert - war in der zweiten Hälfte der 1960er Jahre in sieben Landtage eingezogen. Nach dem Scheitern der Partei bei der Bundestagswahl 1969 an der Fünfprozenthürde (mit 4,3 Prozent) unter ihrem führenden Kopf Adolf von Thadden konnte sie zunächst keine Erfolge mehr verbuchen. Sie blieb in den 1970er und 1980er Jahren in der Regel an der für Parteienfinanzierung wichtigen Hürde von 0,5 Prozent bei Bundestagswahlen bzw. 1,0 Prozent bei Landtagswahlen.

Das sollte sich 2004 ändern. Auf dem Höhepunkt der Kampagnen gegen „Hartz IV“ gelang ihr erstmals wieder der Einzug in ein Landesparlament - in den sächsischen Landtag mit 9,2 Prozent. 2006 kam sie auf 7,3 Prozent in Mecklenburg-Vorpommern. Die NPD hatte sich seit der zweiten Hälfte der 1990er Jahre unter dem Vorsitzenden Udo Voigt mit ihrer Drei-Säulen-Strategie (,Kampf um den Wähler“, „Kampf um die Köpfe“, „Kampf um die Straße“) stark radikalisiert - bis hin zu nationalsozialistischen Tendenzen. Nach dem Erfolg in Sachsen gilt der „Kampf um den organisierten Willen“ als vierte Säule. Die NPD strebt damit das Monopol im „nationalen Lager“ an. Mit ihrem „Deutschlandpakt“ vom Januar 2005 einigten sich NPD und DVU darauf, nicht gegeneinander anzutreten. Zugleich versucht die NPD, militante „Freie Kameradschaften“ zu integrieren. Die aggressiv agierende Partei vertritt im Vergleich zu den 1960er, 1970er und

42 Vgl. die Aufsatzsammlung von Meier, H.: Protestfreie Zonen? Variationen über Bürgerrechte und Politik, Berlin, 2012.

43 Jesse, E.: Demokratieschutz, in: Jesse, E./Sturm, R. (Hg.): Demokratien des 21. Jahrhunderts im Vergleich. Historische Zugänge, Gegenwartsprobleme, Reformperspektiven, Opladen, 2003, 449-474. 
1980er Jahren einen strikt antikapitalistischen Kurs und versucht auf diese Weise, beim „kleinen Mann“ anzukommen. ${ }^{44}$ Die Partei konnte ihre Erfolge bei den Landtagswahlen in Sachsen (2009: 5,6 Prozent) und Mecklenburg-Vorpommern (2011: 6,0 Prozent) wiederholen, wiewohl auf einem niedrigeren Niveau. Freilich schnitt sie bei den Bundestagswahlen 2005 mit 1,6 Prozent und 2009 mit 1,5 Prozent schwach ab. In den neuen Bundesländern ist sie aus sozialisationsgebundenen und situativen Gründen stärker (2009: 3,1 Prozent in den neuen, 1,1 Prozent in den alten Ländern).

Die NPD, die trotz ihres Einzugs in zwei Landesparlamente, trotz der Einbindung der DVU und trotz des Wechsels von Udo Voigt zu Holger Apfel, der ,,seriöse Radikalität ${ }^{\text {“45 }}$ zu propagieren sucht, ist in einem organisatorischen, strategischen, ideologisch und finanziell weitgehend desolaten Zustand. ${ }^{46}$ Gegner, fixiert auf vollmundige Verlautbarungen der Partei, vernachlässigen diesen Gesichtspunkt oft.

An dem harten Extremismus der NPD mit Blick auf Organisation, Strategie und Ideologie - im Vergleich etwa zum weichen Extremismus der Linken ${ }^{47}$ - besteht kein Zweifel: Diese Partei lehnt mit ihren fremdenfeindlichen Ressentiments, rassistischen Stereotypen und ihrer „Volksgemeinschafts“-Ideologie klar die freiheitliche Ordnung ab, dabei strikt antikapitalistisch agitierend. Insofern ist die im Untertitel des Kapitels gestellte Frage bloß rhetorischer Natur.

\section{Verbotsverfahren gegen die NPD?}

Eine Folge der Thüringer Morde war die nahezu reflexhafte Forderung nach einem Verbot der NPD. ${ }^{48}$ Das erste, 2001 von Bundestag, Bundesrat und Bundesregierung gegen die NPD überstürzt eingeleitete Verbotsverfahren endete 2003 mit einem schmählichen Ergebnis für die Antragsteller: Das Bundesverfas-

44 Vgl. Steglich, H.: Rechtsaußenparteien in Deutschland. Bedingungen ihres Erfolges und Scheiterns, Göttingen, 2010; Backes, U./Steglich, H. (Hg.): Die NPD. Erfolgsbedingungen einer rechtsextremistischen Partei, Baden-Baden, 2007.

45 Apfel, H.: Seriöse Radikalität, in: Deutsche Stimme, Nr. 11/2011, 12.

46 Brandstetter, M.: Die „neue“ NPD: Zwischen Systemfeindschaft und bürgerlicher Fassade, Berlin, 2012 (i.E.).

47 Vgl. Jesse, E.: Die NPD und die Linke. Ein Vergleich zwischen einer harten und einer weichen Form des Extremismus, in: Backes, U./Gallus, A./Jesse, E. (Hg.), Jahrbuch Extremismus \& Demokratie, Bd. 21, Baden-Baden 2009, 13-31.

48 Der Spiegel widmete der Verbotsfrage eine Titelgeschichte: Dahlkamp, J. u.a.: Eine unerträgliche Partei, in: Der Spiegel, 13. Februar 2012, 32-41. 
sungsgericht sah in der Vielzahl der V-Männer in den Vorständen der NPD ein unüberwindliches Verfahrenshindernis und stellte das Verfahren ein. ${ }^{49}$ Diese Prozessentscheidung, keine Sachentscheidung, war unvermeidlich. Sie stellte der NPD damit zwar keinen Persilschein aus, doch nahm die streitbare Demokratie Schaden. ${ }^{50}$

Die Politik wollte und konnte sich der öffentlichen erhobenen Forderung nach einem Verbot der NPD aber nicht entziehen. Nachdem die Innenminister aller Bundesländer im März 2012 erklärt hatten, Anfang April alle V-Männer in den Vorständen der NPD ,abzuschalten“, beschlossen die Ministerpräsidenten der Länder Ende März 2012, am 6. Dezember eine Entscheidung für oder gegen ein Verbotsverfahren zu treffen. Die Innenminister sind gehalten, bis Mitte November das Ergebnis ihrer Materialsammlung vorzulegen. Wohlgemerkt: Dabei ist selbst im Fall klarer Beweise nicht gesagt, dass es zu einem Verbotsantrag kommt.

Die Forderung wurde im Wesentlichen mit zwei Argumenten begründet: (Ehemalige) Mitglieder der NPD seien in diese Gewalttaten verstrickt; diese Partei bereite den ideologischen Boden für solche Morde. Aber: Lässt sich Mitwisserschaft und Hilfeleistung Einzelner der Partei als Ganzes zurechnen? Und sind derartige Verbrechen tatsächlich indirekt durch die NPD gespeist, die ja das größte Interesse daran haben dürfte, mit ihnen nicht in Verbindung gebracht zu werden?

Bei der Frage nach einem NPD-Verbot müssen zwei Komplexe voneinander getrennt werden, der deskriptive und der präskriptive. Eine Vermengung ist der Sache nicht zuträglich. Anders formuliert: Ist ein Verbot möglich? Und: Ist ein Verbot nötig? Damit ergeben sich vier Varianten: a) möglich und nötig; b) möglich und unnötig; c) unmöglich und nötig; d) unmöglich und unnötig. Der Verfasser präferiert die Variante b). Die Frage, ob ein Verbot möglich ist, dürfte zu bejahen $\operatorname{sein}^{51}$ - jedenfalls, wenn die Behörden die V-Männer aus den Vorständen der Partei ,abschalten“ und der Antrag „wasserdicht“ ist. Die NPD ist nicht nur eine extremistische Partei, sondern auch eine solche, die aggressivkämpferisch operiert.

49 Flemming, L.: Das NPD-Verbotsverfahren. Vom „Aufstand der Anständigen“ zum „Aufstand der Unfähigen“, Baden-Baden, 2005.

50 Jesse, E.: Der gescheiterte Verbotsantrag gegen die NPD. Die streitbare Demokratie ist beschädigt worden, in: Politische Vierteljahresschrift 44 (2003), 292-301.

51 Weckenbrock, Ch.: Die streitbare Demokratie auf dem Prüfstand. Die neue NPD als Herausforderung, Bonn, 2009. 
Hingegen ist ein solches Verbot wahrlich nicht nötig - sei es aus Gründen der Effizienz (die Fremdenfeindlichkeit verschwindet dadurch nicht), sei es aus Gründen der Liberalität (die Organisationfreiheit ist in einer offenen Gesellschaft ein hohes Gut). Die weitere Radikalisierung der NPD in der zweiten Hälfte der 1990er Jahre erfolgte u.a. aufgrund einer Verbotswelle gegen rechtsextremistische Vereinigungen in der ersten Hälfte jenes Jahrzehnts.

Was die prospektive Seite betrifft, so liegt die Vermutung nahe, dass es nicht zu einem Verbotsantrag kommt. Schließlich wissen Politiker und Sicherheitsbehörden um die Unterwanderung der Partei. Das dem Gericht vorgelegte Material könnte kontaminiert sein. Allerdings ist eine Eigendynamik (wie 2000/2001) keineswegs ausgeschlossen. Freilich dürfte die Politik argumentieren, ein Verbotsantrag sei zwar nötig, aber nicht möglich (wegen der hohen Hürden), um das Gesicht zu wahren. Der Hinweis auf den Europäischen Gerichtshof für Menschenrechte, der im Gegensatz zum Bundesverfassungsgericht auch den Gesichtspunkt der Gefährdung berücksichtigt, zielt in diese Richtung.

Die Grenze bei der Frage nach einem NPD-Verbot verläuft dabei nicht zwischen den Anhängern und den Gegnern der streitbaren Demokratie. Tatsächlich spielt bei der immer wieder entfachten Diskussion viel Symbolpolitik mit. Auf der Seite der Befürworter eines Verbotsantrages stehen zahlreiche Politiker, ${ }^{52}$ die auf diese Weise „Flagge zeigen“ wollen, weil sie wissen, wie populär ein solcher Schritt ist, obwohl er gegen eine geächtete, nicht geachtete Partei kaum notwendig erscheint. Insofern ist die Frage im Unterkapitel mit einem „nein“ zu beantworten.

\section{Abschließende Überlegungen}

Wie ist es um die Akzeptanz der Extremismuskonzeption bestellt, wie um die Konzeption der streitbaren Demokratie? Das Resümee kann nicht nur positiv ausfallen. Zwar halten die tragenden gesellschaftlichen Kräfte daran fest, den parteiförmigen wie den subkulturellen Extremismus zu bekämpfen, doch besteht keine Einigkeit darin, wer als extremistisch zu gelten hat, jedenfalls mit Blick auf die linke Variante, sofern diese weder Gewalt anwendet noch propagiert. Hingegen wird gegen die rechte Variante des Extremismus, ob gewalttätig oder nicht, mitunter in einer Weise vorgegangen, die jakobinischer Natur ist (,keine Freiheit den Feinden der Freiheit").

52 Vor dem Bekanntwerden der rechtsextremistischen Mordserie überwog bei den Vertretern der Unionsund der FDP-Politiker Skepsis gegenüber einem NPD-Verbotsverfahren. 
Die Position, eine Auffassung sei „gefährlich“ und provoziere den „Beifall von der falschen Seite“, verkennt den Sinn wissenschaftlicher Diskurse. Viele Kritiker des Extremismuskonzepts, nicht alle, stehen Strömungen nahe, gegen die sich extremismustheoretische Betrachtungen wenden. Wer diesen vorrangig politische Motivationen unterstellt, argumentiert aus einer „Haltet den Dieb“Reaktion heraus. Die normativ-vergleichende Extremismusforschung wendet sich nicht dagegen, lediglich eine Variante des Extremismus zu untersuchen. Dieser Ansatz ist nicht nur legitim, sondern auch notwendig. Wogegen sie aber Position bezieht, ist die Behauptung, lediglich eine spezifische Richtung lehne den demokratischen Verfassungsstaat ab. Das Verständnis des Grundgesetzes ist der Antiextremismus, der die freiheitliche demokratische Grundordnung schützt.

Wer Politikwissenschaft (auch) als Demokratiewissenschaft versteht, kommt nicht an der Extremismuskonzeption vorbei, ohne deswegen die Plausibilität anderer Ansätze in Zweifel zu ziehen. Der Vergleich gegensätzlicher - und doch verwandter - (tatsächlich oder vermeintlich) antidemokratischer Phänomene ist ein anspruchsvolles Unterfangen, empirisch wie theoretisch. Die Extremismusforschung, die sich an der Universalität der Menschenrechte orientiert, hat keinem „Konjunkturrittertum“, keinen Zeitgeisttendenzen zu frönen. Sie kann und soll in einer demokratischen Gesellschaft nicht Politik mit anderen Mitteln betreiben. Die normative Extremismusforschung darf dem Kampf um politische Deutungshoheiten keinen Vorschub leisten.

Bei der Erörterung des Gefahrenpotentials für den demokratischen Verfassungsstaat spielt nicht nur die Frage der extremistischen Intensität eine Rolle, sondern auch eine Reihe weiterer Faktoren, wie etwa der Erfolg bei Wahlen, die Mitgliederstärke einer Partei, das intellektuelle Potential, die Akzeptanz bei Eliten, die Infiltration in die Mehrheitskultur. Die Frage nach der Gefahr für den demokratischen Verfassungsstaat liegt mithin auf einer anderen Ebene als die Frage nach der extremistischen Intensität einer Organisation oder eines subkulturellen Phänomens.

Heute geht in Deutschland die größte Gefahr für den Bestand des demokratischen Verfassungsstaates wohl nicht von extremistischen Strömungen aus, sondern von der politischen Mehrheitskultur, die ihr politisch-ethisches Koordinatensystem zu verlieren droht. Diese Kritik an der „Mitte“ steht damit in erstaunlicher Nähe zu den Gegnern des Extremismusansatzes. Nur ist sie anders begründet. Die Fundamentalkritik am normativ fundierten vergleichenden Extremismusbegriff ist ein Menetekel. Eine Revitalisierung und Neufundierung des antiextremistischen Gründerkonsenses der zweiten deutschen Demokratie tut not. 
Das gilt für Politik, Publizistik und Wissenschaft gleichermaßen. Durch die gegenwärtige Fixierung auf den „Nationalsozialistischen Untergrund“ wird dies jedoch erschwert. Sie eignet sich bestens zur Instrumentalisierung. ${ }^{53}$

Gewiss, die Extremismusforschung wird in Deutschland bisweilen bekämpft, zum Teil ignoriert, ja tabuisiert, auch wenn die Vielzahl der einschlägigen Periodika und Jahrbücher zu einem gewissen Optimismus Anlass gibt („,Totalitarismus und Demokratie“, „Jahrbuch Extremismus \& Demokratie“, „Jahrbuch für Extremismus- und Terrorismusforschung“, „Jahrbuch Öffentliche Sicherheit“, „Jahrbuch Terrorismus“). Mit Ernst Fraenkel, einem der Gründungsväter der deutschen Politikwissenschaft, gilt: „Eine Politikwissenschaft, die nicht bereit ist, ständig anzuecken, die sich scheuen sollte, peinliche Fragen zu stellen, die davor zurückschreckt, Vorgänge, die Kraft gesellschaftlicher Konvention zur arcana societatis erklärt worden sind, rücksichtslos zu beachten, und die es unterlässt, freimütig gerade über diejenigen Dinge zu reden, über die man nicht spricht, hat ihren Beruf verfehlt. “54

53 Baron, U.: Die Linke und der Rechtsterrorismus, in: MUT, 4/2012, 58-63.

54 So Fraenkel, E.: Die Wissenschaft von der Politik und die Gesellschaft (1963), in: ders. (Hg.), Reformismus und Pluralismus, Hamburg, 1973, 344. 